\title{
The impact of documentation burden on patient care and surgeon satisfaction
}

The views expressed in this editorial are those of the authors and do not necessarily reflect the position of the Canadian Medical Association or its subsidiaries.

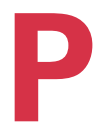

aperwork is commonly defined as "routine work involving written documents such as forms, records, or letters." It is also clear that appropriate documentation (written and/or electronic) is critical to patient safety, continuity of care, and both individual and system quality improvement. ${ }^{1}$ Unfortunately, however, it seems our surgical working lives are increasingly filled with documentation tasks that move us farther away from high-quality bedside patient care and instead toward becoming efficient scribes recording preceding medical events.

The transition to electronic health records (EHRs; by 2015, 92\% of hospital-based physicians were using EHRs $^{1}$ ) has been both a tremendous blessing and a challenging curse. It is evident EHRs are the future of our discipline. They have the potential to interface with artificial intelligence in a way that only science fiction has previously predicted. They improve the quality of health care, diagnostic accuracy, patient safety, continuity of care, outcomes and performance tracking. In some of our best examples, EHRs enhance efficiencies in patient flow and in billing/reimbursement by reducing redundancies and delays. Unfortunately, these positive traits have also been seeded with unintended consequences, including prolonged surgeon work hours, frustration with repeatedly changing information systems, and a decrease in the time available to actually care for patients at the bedside. This "documentation burden" has been shown to be associated with increased medical errors, documentation mistakes, patient safety concerns, job attrition and emotional despair among health care providers. ${ }^{1}$ Considering physicians now spend up to twice as much time completing electronic documentation and administrative tasks $(50 \%)$ as time engaging in direct bedside patient care $(12 \%-27 \%)$, it is not surprising that surgeon discontent has reached an all-time high..$^{2-4}$ No one, to our knowledge, pursues medical school training to become experts in documentation especially at a $2: 1$ ratio with patient care.

Burden is defined as "a duty or responsibility that causes worry, difficulty, or hard work." This concept is structurally distinct from burnout. ${ }^{5}$ Given the recent focus on physician mental and physical wellness, sur- geons must genuinely pause to contemplate their own documentation burden (i.e., within their individual practice/health care system). An honest appraisal of its potential impact on their own burnout potential and quality of life is a necessary assessment.

While we typically contemplate the documentation burden associated with clinical care, there has also been a tremendous rise in mandatory assignments among other domains within many of our practices. Examples include research (university ethics review boards and processes; provincial health care research impact assessments; individual program barriers and obstacles; grant agency applications), physician reimbursement (billing documentation; time stamp confirmations; fees submission; shadow-billing), teaching (competency-based education assessments; trainee evaluations), professional development (regulatory body performance, competency assessments and renewal exams; professional surveys) and quality improvement (introduction of new technologies, processes, pathways, checklists, safety protocols, and programs; safety learning incident reviews). Although these examples may consume varying amounts of time and energy in a given surgeon's practice, they are defined by a common link: lack of direct physician-patient engagement. This is also relevant to our education of medical students and residents, as historical bedside teaching rates of $75 \%$ now approximate $8 \%-19 \%$ depending on the medical school. ${ }^{1-4}$ While many of us who completed components of our training (and/or worked) in the United States were mesmerized by the amount of time faculty surgeons were engaged in physical documentation (both written and electronic; i.e., daily formatted progress notes, admission records, discharge summaries, insurance updates, billing code adjustments), the truth is that we all thought to ourselves "thank goodness we don't have to do this in Canada." The reality is that now we do. Upon deeper contemplation, our practices have become very similar with regards to documentation burden.

Interestingly, we can also look to our southern colleagues for potential solutions. In a number of highprofile surgical subspecialty areas, our American contemporaries have employed scribes who complete the 
vast majority of their required documentation on behalf of surgeons. These surgical practices also frequently include nonphysician clinical help in a 1:1 ratio for high relative value unit (RVU) surgeons (i.e., clinically busy). Associate health care providers (nurse practitioners, physician assistants, clinical associates, surgical hospitalists) not only contribute to direct patient care, but also to documentation requirements. While our initial response in pondering this working environment is envy, we should be cognizant that these resources are in fact used in practice models within Canada as well. The most notable examples would be some of our medical counterparts (who are often salaried, as opposed to feefor-service). Within surgery, many high-volume ophthalmology outpatient practices employ dictation scribes, documentation specialists, billing (public and private) experts, and other clinical specialists (e.g., optometrists) to increase throughput, enhance efficiency, and focus the surgeon/physician on direct patient care. This clearly requires a significant shift in many of our practice models, funding sources and conceptual framework, but perhaps is inevitable to ensure improvements in satisfaction among future generations of surgeons. We must remember however, that in the end, personal accomplishment and pride arises from providing superb patient care, not empty email inboxes and zeroed medical record incompletions.

Chad G. Ball, MD, Paul B. McBeth, MD, MASc
Affiliations: From the University of Calgary, Calgary, Alta. (Ball); Alberta Health Services, Calgary, Alta. (McBeth); and coeditor-in-chief, Canadian Fournal of Surgery (Ball).

Competing interests: None declared.

Content licence: This is an Open Access article distributed in accordance with the terms of the Creative Commons Attribution (CC BYNC-ND 4.0) licence, which permits use, distribution and reproduction in any medium, provided that the original publication is properly cited, the use is noncommercial (i.e., research or educational use), and no modifications or adaptations are made. See: https://creativecommons. org/licenses/by-nc-nd/4.0/

DOI: 10.1503/cjs.013921

\section{References}

1. Moy AJ, Schwartz JM, Chen R, et al. Measurement of clinical documentation burden among physicians and nurses using electronic health records: a scoping review. $7 \mathrm{Am}$ Med Inform Assoc 2021;28:998-1008.

2. Poissant L, Pereira J, Tamblyn R, et al. The impact of electronic health records on time efficiency of physicians and nurses: a systematic review. 7 Am Med Inform Assoc 2005;12:505-16.

3. Colicchio TK, Cimino JJ. Clinicians' reasoning as reflected in electronic clinical note-entry and reading/retrieval: a systematic review and qualitative synthesis. 7 Am Med Inform Assoc 2019; 26:172-84.

4. Grant P. Physician job satisfaction in New Zealand versus the United Kingdom. N Z Med f 2004;117:U1123.

5. Jane Lemaire on burnout, resiliency and coping during COVID. Cold Steel [Podcast]. Available: https://soundcloud.com/cjs -podcast/e19-jane-lemaire-on-burnout-resiliency-and-coping -during-covid (accessed 2021 May 6). 